Recherches en didactique des langues et des cultures

Les cahiers de l'Acedle

13-3 | 2016

Travailler sur et à partir de textes écrits en classe de langue étrangère

\title{
Des lectures et des écritures du texte littéraire en langue étrangère
}

Victoria Béguelin-Argimón

\section{OpenEdition}

1 Journals

Édition électronique

URL : http://journals.openedition.org/rdlc/1290

DOI : $10.4000 /$ rdlc. 1290

ISSN : 1958-5772

Éditeur

ACEDLE

Référence électronique

Victoria Béguelin-Argimón, « Des lectures et des écritures du texte littéraire en langue étrangère »,

Recherches en didactique des langues et des cultures [En ligne], 13-3 | 2016, mis en ligne le 31 décembre 2016, consulté le 30 avril 2019. URL : http://journals.openedition.org/rdlc/1290 ; DOI : 10.4000/ rdlc. 1290

Ce document a été généré automatiquement le 30 avril 2019

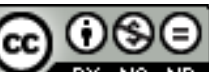

Recherches en didactique des langues et des cultures is licensed under a Creative Commons AttributionNonCommercial-NoDerivatives 4.0 International License 


\title{
Des lectures et des écritures du texte littéraire en langue étrangère (LE)
}

\author{
Victoria Béguelin-Argimón
}

\section{Introduction}

1 Très récemment, l'écrivain péruvien Mario Vargas Llosa avouait qu'il avait été marqué, dès son enfance, par ses premières lectures de romans d'aventures : au travers de la fiction, ces récits lui avaient permis non seulement de se déplacer dans l'espace et dans le temps mais aussi d'incarner les destinées et les expériences d'une énorme variété de personnages. Et il soupçonnait fortement ce moment d'être à l'origine de son besoin - ou de son appétit - pour l'écriture, ses premières incursions comme écrivain en herbe étant le divertissement d'un enfant qui se plaisait à amender ou à inventer une suite aux histoires qu'il jugeait trop courtes. Avec ces mots, Vargas Llosa faisait état de son vécu personnel - subjectif, participatif et affectif - en tant que lecteur, ainsi que de ses précoces interventions scripturales dans les textes d'autrui ${ }^{1}$. De quoi ébranler deux visions encore enracinées lorsqu'il s'agit d' " enseignement » de la littérature en contexte scolaire : d'une part, celle de considérer que plus la lecture est distancée et basée sur une posture analytique plus elle est valable et, d'autre part, celle de concevoir le texte littéraire comme un objet intouchable, fini, clos.

2 Effectivement, depuis une dizaine d'années, trois problèmes ont été décelés concernant la lecture des textes littéraires en L1 en contexte scolaire que J. David (David, 2014 : 23) résume ainsi : un "[f]ormalisme procédurier - dommageable pour la quête d'un sens humaniste ; exception littéraire - et exclusion de la littérature de la sphère des expériences ordinaires ; passivité des élèves - et inhibition de tout engagement individuel dans la lecture et l'interprétation collective des textes littéraires ». En réponse à ces constats, de nouvelles approches à la littérature en L1 ont souligné l'importance de prendre en 
compte, d'une part, la notion de «texte du lecteur » (Mazauric, Fourtanier \& Langlade, 2011a et 2011b), prônant qu'on valorise l'activité des lecteurs dans la reconfiguration des œuvres, donc leur apport à la réalisation de l'œuvre lue ; d'autre part, l'engagement personnel des apprenants dans l'acte de lire, ce qui présuppose, entre autres, l'entrée dans la sphère de l'enseignement de la lecture dite "ordinaire", donc subjective, participative et affective, trop souvent bannie des contextes scolaires ou académiques (Rouxel, \& Langlade, 2004 et Baroni \& Rodriguez, 2014) ; enfin, troisième notion portée au-devant de la scène, celle des "écritures de la réception", à savoir l'exploitation de l'ensemble des productions graphiques ou iconiques qu'un lecteur peut produire face à un texte littéraire (Le Goff, 2011). Ces approches, proposées comme portes d'accès à la lecture littéraire en L1, peuvent s'avérer également très fécondes lorsqu'il s'agit de la lecture de textes littéraires en LE.

3 En fait, la démarche actionnelle et participative dans l'enseignement des langues étrangères prônée par le Cadre européen commun de référence pour les langues (2001) présente bien des points communs avec les approches au texte littéraire en L1 qu'on vient d'évoquer. Certes, le CECR ne s'occupe pas de la lecture des textes littéraires - tout autant qu'il ne s'occupe pas de comment aborder la lecture d'un autre genre quelconque de discours -, mais il considère les apprenants comme des acteurs sociaux ayant des tâches à accomplir. Celles-ci doivent les impliquer le plus possible - en incluant des échanges et des activités personnalisées qui sollicitent leurs avis individuels et leurs vécus - et se veulent particulièrement bénéfiques pour l'augmentation de leur motivation, pour leur investissement dans l'apprentissage et, à terme, pour leur utilisation de la LE. C'est dans cette confluence des principes du CECR et des recherches sur l'approche au texte littéraire en L1 que de nouveaux accès à la lecture littéraire en LE peuvent être explorés, par le biais d'une grande variété d'activités langagières, tant orales qu'écrites qui, tout en augmentant le capital linguistique de l'apprenant, construisent sa compétence communicative, socio-culturelle et littéraire ainsi que ses stratégies de lecteur, et mettent également à plat ses difficultés face au texte littéraire ${ }^{2}$.

Cet article s'interrogera dans un premier temps sur les écueils particuliers du texte littéraire pour un lecteur de LE et ébauchera ensuite des voies susceptibles de faciliter l'accès à des textes littéraires longs, tels les romans, par l'établissement d'un dialogue entre le texte et le lecteur - surtout moyennant l'écriture - et par l'introduction de la lecture dite ordinaire en classe. Nous illustrerons nos propositions par des exemples tirés d'une séquence didactique conçue pour la lecture du roman El lápiz del carpintero de l'écrivain espagnol Manuel Rivas (Rivas, 2006) dans une classe d'espagnol LE.

\section{Spécificités de la lecture littéraire en LE}

5 On connait bien les écueils que présente la lecture en LE pour les apprenants : il s'agit très souvent d'une lecture linéaire, plus lente qu'en L1, enrayée tant par le manque de vocabulaire et par des connaissances grammaticales limitées, que par l'application de stratégies de lecture inadéquates ou restreintes, ce qui a comme conséquence que le décodage l'emporte, parasite la compréhension et empêche l'interprétation ${ }^{3}$.

6 En ce qui concerne, plus spécifiquement, la lecture de textes littéraires, les difficultés s'accroissent : trop souvent, certains apprenants ne sont que des lecteurs occasionnels de littérature dans leur propre langue et leur «compétence littéraire»- limitée aux connaissances scolaires acquises en L1 - est peu développée. En outre, des 
représentations généralisées sur la littérature peuvent provoquer des blocages car l'accès à celle-ci peut être perçu comme le couronnement d'un long et pénible apprentissage langagier où la maitrise de la langue est la conditio sine qua non pour la compréhension et la jouissance du texte littéraire. L'aura d'autorité dont la littérature est investie socialement impose, en outre, un certain respect aux apprenants, les déstabilise et ébranle leur confiance.

7 Aux problèmes liés tant à la lecture en général qu'aux ressentis face à la littérature, viennent s'ajouter les difficultés intrinsèques au texte littéraire : compréhension de l'organisation du discours (structure, temps de l'énoncé/temps de l'énonciation), de l'intrigue, des personnages, du rôle du narrateur, des voix narratives, du point de vue, de la typologie du texte et des séquences textuelles, du contexte, etc. Le travail sur la construction du sens à partir de l'implicite et du non-dit, qui est demandé au lecteur du texte littéraire, est bien plus important que dans n'importe quel autre type de texte. A toutes ces difficultés, se greffent l'ironie, l'humour, l'ambigüité, une langue polysémique et des images de style, propres au fait littéraire ; enfin, les références culturelles ou l'intertextualité peuvent brouiller définitivement l'interprétation du message.

\section{Revaloriser la lecture ordinaire en LE}

\section{Conférer une dimension communicative, interprétative et créative à la lecture littéraire}

8 Les approches didactiques habituelles du texte littéraire en LE, contribuent-elles à réduire les problèmes évoqués? Pas vraiment, car bien souvent la lecture littéraire en classe de LE oscille entre deux pôles : celui de considérer le texte littéraire comme un simple moyen d'améliorer les compétences linguistiques des apprenants et celui de considérer la lecture analytique comme seule possibilité de lecture littéraire digne de ce nom. On tend à mettre de côté toute valorisation de la découverte individuelle et des réactions subjectives et affectives, caractéristiques de la lecture ordinaire. Lors de la lecture d'un récit, l'immersion dans l'univers fictionnel, sensoriel ou symbolique, l'acceptation de l'«illusion référentielle ", l'intérêt pour l'intrigue, l'empathie pour les personnages (David, $2014: 24-25$ ), bref tout ce qui peut faire la force du texte littéraire est considéré comme un passage obligé - un moindre mal en quelque sorte -, pour l'accès à une lecture "sérieuse ", à la lecture dite lettrée, à la "vraie » lecture littéraire. Or, si comme nous l'avons mentionné, les nouvelles approches à la lecture littéraire en L1 redonnent de l'importance à la lecture ordinaire, au «texte du lecteur » et - quoique moins novateur - à la manipulation du texte moyennant l'écriture comme outils précieux pour l'accès au texte littéraire, il faut se persuader que ce sont également les plus puissants moteurs pour la lecture littéraire en LE.

9 En travaillant avec des romans en LE, par exemple, l'enseignant peut être tenté de jouer le rôle de «fournisseur d'informations » sur l'auteur, le contexte historique de l'ouvrage, le mouvement littéraire, les enjeux de la structure, les difficultés posées par les voix narratives, etc., et il peut être également incité à expliquer le texte, à donner des interprétations, ou encore à proposer des pratiques voisines à l'analyse textuelle, ce qui peut s'avérer particulièrement éprouvant pour les lecteurs de LE. Or la mise en place de parcours de lecture et d'activités appropriés permet de passer le témoin à l'apprenant pour qu'il adopte un rôle actif dans la découverte de la complexité du texte littéraire. 
10 Effectivement, si on veut faire de la lecture littéraire un acte communicatif, il faut: 1. un texte avec lequel on entre en dialogue, qu'on interroge, qu'on essaye de comprendre, face auquel on réagit ; 2 . des personnages avec qui on entre en dialogue, à qui on écrit, à qui on parle, qui parlent entre eux ; et 3. des auteurs avec qui on entre en dialogue pour leur poser les questions suscitées par le texte.

11 Si on veut en faire un acte interprétatif, il faut : 1. appréhender la spécificité des textes littéraires ; et 2. être un lecteur-détective, perspicace, chercheur.

12 Enfin, si on veut en faire un acte créatif, il faut un texte qu'on puisse amender et modifier. Comme le soutiennent M.-C. Albert et M. Souchon, «[p]our que la communication puisse s'établir entre le texte et le lecteur il faut que celui-ci puisse s'y introduire et intervenir. Prendre la place du scripteur et anticiper et modifier le texte d'auteur permet d'éviter les blocages tenant à cette représentation de la littérature et rétablit la circulation de la communication littéraire» (Albert \& Souchon, 2000: 62). Effectivement, les activités faisant le pont entre lecture et écriture permettent d'affronter les différents enjeux du texte littéraire, de réaliser ses propres interprétations et d'accéder ainsi à des niveaux supérieurs de lecture. Si l'on en croit l'exhortation de Ricardou (1990: 108) «[d]eviens, lecteur, le scripteur que tu es en puissance, si tu veux davantage comprendre, et, du coup, mieux réussir l'acte même auquel tu prétends " - , les activités d'écriture qui ont comme point de départ les textes littéraires offrent une voie privilégiée mettant en avant la dimension interactive du texte, favorisant la reconstruction de son sens, et a fortiori, contribuant à l'émergence du "texte du lecteur», et permettant enfin un engagement personnel dans la lecture.

\section{Lire pour le plaisir, choisir ses textes}

Si nous nous remontons au premier acte individuel et personnel face à une éventuelle lecture - l'élection du texte à lire - nous pouvons nous demander si un tel choix est possible pour les apprenants en contexte scolaire, surtout lorsqu'il s'agit d'une lecture en LE. On sait bien que l'école reste un espace "à part " qui ne saurait remplacer ou contrefaire le contexte "hors école", mais on peut néanmoins créer des situations les plus authentiques possibles pour rapprocher la lecture en classe de la lecture dite ordinaire. En général, le choix de la lecture relève de l'enseignant - on invoque des raisons pratiques - mais il y a toutefois des moyens d'y impliquer les apprenants avec des activités qui fonctionneront comme un travail de pré-lecture. Le simple fait d'être engagé dans le choix d'un texte dans lequel on devra s'investir pendant plusieurs semaines, aura comme conséquence de créer des expectatives chez les futurs lecteurs et d'augmenter leur motivation ; en même temps, les activités proposées peuvent donner quelques connaissances de base sur les potentiels textes à lire, activer des connaissances, filtrer des difficultés linguistiques et fournir des informations contextuelles pour la lecture à entreprendre.

Multiples sont les activités qu'on pourrait présenter pour effectuer le choix d'un roman à lire en classe et nous en évoquerons ici quelques-unes ; pour que la tâche reste faisable, l'enseignant peut sélectionner préalablement cinq ouvrages parmi lesquels l'élection doit être portée.

1. Lecture comparée des quatrièmes de couverture des romans proposés : on donnera à lire ces textes, photocopiés, afin de compléter un tableau comportant des données telles que le titre de l'ouvrage, le nom des personnages, des détails sur ceux-ci, le lieu et le temps de l'action, 
le thème, et les particularités du texte. Une case sera réservée pour les commentaires de l'apprenant concernant ses préférences.

2. Lecture de critiques des romans provenant de la presse digitale ou de blogs privés : on procédera comme dans le cas précédent, en faisant remplir à l'apprenant le même type de tableau synoptique.

3. Lecture d'extraits choisis des romans : cette activité permet non seulement de se faire une idée de la manière dont le livre est écrit mais, surtout, aiguise la curiosité des lecteurs potentiels. Le choix des passages sera axé sur des moments de rupture, des moments forts de l'intrigue qui laisseront le lecteur avec une envie d'en savoir davantage et ils seront lus à l'aide d'un tableau synoptique comme celui décrit sous 1. L'emploi d'un tel tableau permet de rassembler les informations de manière synoptique en donnant la possibilité d'établir rapidement des rapports entre les différents romans ainsi que d'effectuer des comparaisons.

4. Observation des couvertures des romans : le travail part ici de l'image et vise à créer des anticipations et des hypothèses sur différents aspects du texte tels que l'époque et les lieux de l'action, les personnages ou le thème.

5. Visualisation de séquences d'adaptations cinématographiques des romans, s'il y en a, avec des activités similaires à celles proposées pour la lecture des extraits.

6. Exploitation des book-trailers (vidéos promotionnels combinant images, son et paroles) : les maisons d'édition ont de plus en plus recours à ce moyen de promotion de leurs publications et, quoique parfois très pauvres du point de vue informatif, dans bien des cas la richesse et variété des données fournies s'avère d'une grande utilité pour aider à porter un choix sur un ouvrage à lire.

7. Lecture des pages Internet d'un auteur : parfois très complètes, elles apportent, entre autres, des éléments biographiques, aujourd'hui très souvent axés sur une image de l'écrivain faisant une large place à la dimension personnelle (par exemple, inclusion de photos concernant sa vie privée ou expression d'opinions personnelles sur des sujets très divers). Le sentiment d'établir un lien individuel avec l'auteur éveille chez le futur lecteur une complicité susceptible de l'amener à vouloir en savoir davantage sur l'écrivain ou sur sa production, et à porter son choix sur un de ses ouvrages. En outre, ces pages rassemblent des critiques littéraires, des commentaires des livres, des entretiens audio ou vidéo et des hyperliens vers d'autres pages. Les sites les plus riches contiennent de courtes vidéos où l'auteur fournit des explications sur l'intrigue, les personnages, le contexte historique ou le contexte de création de tel ou tel ouvrage.

15 Pour dynamiser les activités précédentes il conviendrait d'organiser le travail par groupes de cinq apprenants et, au lieu de demander à chacun de lire toutes les quatrièmes de couverture ou toutes les critiques, par exemple, il lirait une des pièces proposées et en ferait ensuite un rapport aux autres membres du groupe afin qu'ils puissent compléter leurs tableaux et se faire une idée des cinq possibilités de lecture. Une fois en possession de toute l'information, les apprenants argumenteraient leurs choix.

Il s'agit, dans tous ces cas, d'activités et de supports qui peuvent être combinés les uns avec les autres et qui renforcent bien évidemment les compétences langagières facilitant l'entrée dans les romans proposés par de multiples voies parallèles. En outre, ces activités portent les apprenants à discuter et à négocier avec leurs pairs du choix final de lectureil faut motiver les raisons des préférences (pourquoi je veux lire tel roman ou pourquoi pas), à définir un objectif de lecture (dans quel but je veux lire ce texte) - ainsi qu'à générer des expectatives. En effet, ce sont des activités qui, en fournissant une information incomplète, éveillent la curiosité et suscitent des questions telles que : que m'apportera ce texte?, qu'est qu'il signifie?, etc. 
17 Mais ces activités contribuent également à l'appropriation des compétences littéraires car l'apprenant est emmené, par exemple, à reconnaitre un genre textuel et à se prononcer sur lui (j'aime / je n'aime pas et pourquoi), à travailler sur l'intertextuel (d'autres textes portant sur le même sujet) ou à devenir conscient des stratégies de compréhension écrite à développer, en anticipant les problèmes qu'une lecture donnée pourrait poser. Loin d'être une simple « mise en bouche », elles contribuent à l'autonomie des lecteurs.

\section{Entrer dans El lápiz del carpintero de Manuel Rivas}

Nous illustrerons quelques-unes des propositions évoquées ci-dessus, notamment la mise à profit des synergies entre lecture littéraire et écriture, en présentant quelques activités tirées d'une séquence didactique créée pour la lecture en espagnol du roman de Manuel Rivas El lápiz del carpintero. Ces activités sont destinées à des apprenants d'espagnol de niveau B1 consolidé ou B2 et elles ont été testées avec des étudiants de première année de la Section d'espagnol de l'Université de Lausanne. Il est important de signaler la langue de scolarité des apprenants, le français, et la LE, l'espagnol, car sans doute faut-il compter ici sur l'intercompréhension entre ces deux langues romanes pour proposer une lecture aussi exigeante.

Le roman présente tout au long de ses sept premiers chapitres un écueil important lié, notamment, à sa structure temporaire et aux voix narratives. Les deux premiers chapitres se situent à la fin de la vie des deux personnages principaux : le premier, le médecin Daniel Da Barca, ancien militant républicain qui a survécu à des pelotons d'exécution, à des peines de mort, au bagne et enfin à l'exil pendant la guerre civile espagnole et la période franquiste ; le deuxième, Herbal, son gardien de prison et son bourreau qui, d'un bout à l'autre du roman, racontera la vie de Da Barca, intimement entrelacée à la sienne, à une jeune prostituée. Après les deux premiers chapitres situés dans les années 1990, le récit remonte à l'été 1936, début de la guerre civile espagnole. De multiples voix narratives s'entremêlent tout le long du récit mais laissent ressortir celle de l'Herbal narrateur, s'exprimant parfois à la première personne, celle d'un narrateur à la troisième personne, focalisé sur Herbal, et celle d'un narrateur omniscient, aussi à la troisième personne. Et il faut attendre jusqu'au septième chapitre pour prendre connaissance d'un élément essentiel de la trame: l'amour qu'Herbal, issu d'une plus que modeste famille du monde rural, porte depuis enfant pour la riche et belle Marisa Mallo, devenue la fiancée de Da Barca.

La complexité du début du roman peut dérouter des apprenants de LE car il faut se familiariser avec les personnages, prendre conscience du flash-back, identifier les voix narratives et saisir le principal mobile qui mène Herbal à persécuter Daniel Da Barca. Par contre, la division du roman en chapitres, parfois assez brefs et présentant une certaine autonomie - quelques-uns peuvent se lire comme des récits indépendants -, est un élément qui facilite la lecture et qu'on peut exploiter pour appréhender le roman avec plus d'aisance. Dans tous les cas, une séquence didactique comprenant, dans un premier temps, une lecture guidée et un travail avec les textes du lecteur et, dans un deuxième temps, une lecture cursive, s'avère très porteuse. 


\section{Des activités guidées : dialoguer avec le texte par l'écriture}

21 Pour entrer dans la lecture de ce roman nous avons tracé un itinéraire particulier qui commencera, en guise d'amorce, par la lecture du chapitre 7. Le but est de faire pénétrer le lecteur dans le roman et de le familiariser avec les personnages pour qu'il comprenne un des principaux moteurs de la trame. Il s'agit d'un chapitre de trois pages, qui peut même fonctionner comme un bref récit indépendant. La lecture de l'extrait permet, en outre, tant de se faire une idée de la manière dont le roman est écrit que d'aiguiser la curiosité du lecteur potentiel en l'incitant à vouloir en savoir davantage. Voici les activités proposées :

Fiche 1

Lis le chapitre 7 du roman de l'écrivain espagnol Manuel Rivas, El lápiz del carpintero.

Dès que tu l'auras fait, essaye de tracer avec ton/ta camarade le profil d'Herbal en te basant sur ses souvenirs d'enfance :

Quels détails nous indiquent la classe sociale à laquelle il appartient, ses liens avec les autres membres de sa famille (sa sœur, son père), sa vision de la vie, son rapport avec Marisa Mallo?

Est-ce que tu penses que c'est un enfant sensible?

Comment imagines-tu ce personnage à l'âge adulte, alors qu'il a eu une enfance comme celle qu'on devine dans ce bref récit?

Esquisse le profil de Marisa Mallo. Quelles informations fournit le texte au sujet du personnage?

Les vies des trois personnages qui apparaissent dans ce récit ont été unies jusqu'à leur mort.

Pourquoi? Comment? Si tu devais écrire un roman sur eux, quelle histoire imaginerais-tu?

Propose une trame possible par écrit.

Les questions 2 et 3 visent à une reconstruction de la personnalité de deux des personnages principaux et de leur évolution tout au long du roman ainsi qu'une prise de position des lecteurs vis-à-vis d'eux. La question 4 permet de formuler des hypothèses de lecture, moyennant un bref travail d'écriture sur le rapport des personnages entre eux et le fil qui les unira tout au long de leurs vies. Il s'agit de créer des scénarios qui anticipent la configuration de l'intrigue et qui contribuent à faciliter la lecture. Nous ne nous attendons pas, bien entendu, à obtenir des histoires qui collent au texte cible mais plutôt à la construction d'un bref récit cohérent et vraisemblable par rapport à la situation de départ.

La suite de la séquence propose un travail ayant comme objectif de se représenter globalement le roman, ses personnages, les principaux conflits, le contexte historique et géographique dans lequel se déroule l'histoire ainsi que certaines caractéristiques du texte en lui-même. Pour cette partie de l'activité nous proposons un travail en binôme : un apprenant lit une quatrième de couverture et l'autre une critique littéraire, dans ce cas tirée d'un blog afin de compléter le tableau suivant avec les données fournies par son texte. Ensuite, ils échangent l'information dont ils disposent pour essayer de réunir un maximum de renseignements dans le tableau ${ }^{4}$. 


\begin{tabular}{|l|l|l|l|l|}
\hline Fiche 2 & $\begin{array}{l}\text { Époque } \\
\text { ambiance de } \\
\text { l'époque }\end{array}$ & $\begin{array}{l}\text { Nom des } \\
\text { personnages } \\
\text { Dersonnages / faits } \\
\text { importants de leurs vies }\end{array}$ & $\begin{array}{l}\text { Lieu de } \\
\text { l'action }\end{array}$ & $\begin{array}{l}\text { Caracteristiques du } \\
\text { texte / Opinions }\end{array}$ \\
\hline & & & & \\
\cline { 2 - 5 } & Prisonniers & & & \\
\hline & Zalo Puga & & & \\
\hline & $\begin{array}{l}\text { Le crayon du } \\
\text { charpentier }\end{array}$ & & & \\
\hline
\end{tabular}

Un aspect intéressant à noter est que, afin d'introduire la lecture d'un texte littéraire par le biais des critiques littéraires, l'enseignant pourrait être tenté de se référer exclusivement aux textes publiés dans la presse de qualité et signés par des critiques littéraires reconnus. Or, ces documents sont rarement de vraies portes d'accès aux textes littéraires pour des lecteurs d'une LE car ils reflètent, presque uniquement, une lecture savante, très éloignée de celle que l'apprenant sera capable de mener à bien. Par contre, Internet regorge de «textes de lecteurs » qu'on trouve dans la profusion de blogs privés consacrés à la littérature et dans lesquels les lecteurs s'expriment en toute liberté sur leurs lectures. Ces textes s'avèrent d'une grande utilité en classe de LE puisqu'ils contiennent des réactions très spontanées sur les textes et qu'ils verbalisent souvent des difficultés de lecture ou apportent des commentaires sur les stratégies adoptées par les lecteurs pour lire tel ou tel ouvrage. Ils rassemblent, en plus, les commentaires d'une communauté de lecteurs qui offrent une pluralité de visions sur l'ouvrage. Avant de s'engager dans une lecture, il est utile pour le lecteur de LE de savoir qu'aborder tel ou tel texte peut ne pas être aisé et de voir comment un lecteur, même de L1, s'est attelé à la tâche. Voici ce qu'on lit, par exemple, dans la critique fournie aux apprenants pour cette séquence :

Le début du livre m'a sonnée, m'a décentrée et j'ai dû recommencer la lecture. Arrivée au même point, je me suis à nouveau fourvoyée dans l'histoire étant donné que le récit change de perspective et ce changement se produit brusquement, sans qu'on en soit averti ${ }^{5}$.

Mais la lectrice ajoute aussi pourquoi il valait la peine de lire le roman :

Ce livre, en plus de me distraire et de m'enchanter par la façon dont il est écrit, m'a servi pour approfondir mes connaissances sur la guerre civile, ses haines et ses secrets.... J'ai découvert beaucoup de choses que j'ignorais auparavant [...] J'ai ressenti de l'empathie pour le docteur Da Barca et moins pour Herbal parce qu'il est un homme rustre et méchant, avec toutefois des touches de bonté que l'envie cache à maintes reprises ${ }^{6}$. 


\section{Fiche 3}

1. Nous nous trouvons dans les années 90 du XXe siècle. Daniel Da Barca a réussi à survivre à toutes les difficultés de la guerre civile espagnole et vit maintenant en Galice avec son épouse Marisa Mallo. Tu es un journaliste et ton journal t'a chargé d'écrire une interview avec cet homme qui symbolise la résistance contre le franquisme. Quel âge a-t-il, à ton avis? Prépare l'entretien. Imagine dix questions que tu aimerais lui poser. Écris-les.

2. Lis ensuite le premier chapitre du roman. Tu verras que le journaliste Carlos Sousa se rend chez Daniel Da Barca pour l'interviewer alors que celui-ci est déjà très âgé et qu'il est mourant. Après la lecture du premier chapitre, vérifie si toutes tes questions ont obtenu une réponse. Si ce n'est pas le cas, tu arriveras probablement à compléter l'information au fur et à mesure que tu avanceras dans la lecture du roman. Garde ces questions et essaye d'obtenir des réponses.

3. Après la lecture du premier chapitre : avec tout ce que tu sais maintenant sur Daniel Da Barca écris le reportage que ton journal t'a demandé.

La consigne de la première activité fournit par elle-même quelques éléments du contexte dans lequel se situe le début du récit, ce qui facilite d'emblée la tâche de lecture. Ensuite, l'activité repose sur le principe de l'anticipation : l'apprenant doit se muer en journaliste et imaginer ce qu'il aimerait connaitre sur le personnage principal. Les questions fonctionnent autant comme grille de lecture que comme horizon d'attente. L'activité 2 propose un travail de vérification des questions que l'apprenant s'était posées ainsi qu'un réservoir de questions momentanément sans réponse qui vont guider sa lecture tout au long de la lecture du roman. Enfin, le l'écriture du reportage demandé dans l'activité 3 vise, d'une part, à ce que l'apprenant, en revenant sur le chapitre, le relise pour bien le comprendre et pour pouvoir ainsi en tirer toutes les informations nécessaires à l'écriture du texte journalistique. La relecture que l'apprenant doit faire peut être considérée comme "naturelle", ayant un but très précis, car il a besoin d'un maximum d'informations s'il veut produire le meilleur reportage possible. D'un autre côté, le travail vise à la production d'un texte de genre différent au texte de départ ce qui implique une reformulation des contenus avec une autre structure et un autre point de vue.

D'autres activités diverses - non basées sur des réécritures -, portant sur le travail des voix narratives et sur le contexte historique, sont proposées jusqu'au chapitre 7 aidant l'apprenant à se former une idée complète des enjeux du roman. À partir de ce chapitre, l'apprenant est armé face aux principaux écueils du texte et on lui propose des moments de lecture individuelle. 


\section{Une activité libre : la lecture cursive} lecture «ordinaire » (vs lecture savante ou lettrée), connue aussi comme lecture «en
progression» (vs lecture en compréhension) ou " cursive» / « extensive » (vs lecture analytique $)^{7}$. Si la réhabilitation de ce mode de lecture en L1 en contexte scolaire est revendiquée aujourd'hui, il doit l'être tout autant quand il s'agit de lecture en LE. On sait que pour l'apprentissage d'une langue, c'est la fréquentation des mots, des structures morpho-syntaxiques, des formes propres à la langue ce qui permet de progresser. La lecture guidée et accompagnée, comme on l'a proposée pour les premiers chapitres du roman, est indispensable en LE mais doit se combiner avec des moments de lecture libre, d'exposition au texte suivi où le lecteur est maitre de le parcourir comme bon lui semble, de s'arrêter, de reprendre la lecture, de se retrouver en tête-à-tête face au texte, même au risque de ne pas « tout saisir ».

C'est une lecture que l'apprenant arrêtera autant de fois qu'il le voudra pour se poser tout autant de questions. La lecture ordinaire sollicite le lecteur tout autrement car il doit puiser dans sa mémoire, son vécu, son bagage culturel, sa personnalité et ses sentiments. Or, une différence entre la lecture en L1 et la lecture en LE est que dans celle-ci, il convient de fournir des outils pour faire émerger les processus de lecture sous-jacents. Dans ce cas, quelques questions accompagnant cette lecture individuelle pourraient être posées:

1. Ai-je déjà lu quelque chose de semblable?

2. Ai-je déjà expérimenté, connu quelque chose de semblable?

3. A quoi ça se rapporte?

4. Comment je réagis/je réagirais?

5. Qu'est-ce que je ressens?

6. Qu'est-ce qui m'émoi?

2 C'est à ce stade-là qu'on peut également fournir des objectifs bien précis pour poursuivre la lecture cursive du roman. Quelques idées possibles seraient :

Choisis une des activités suivantes que tu devras réaliser une fois le roman terminé. Pendant ta lecture, tu devras récolter les informations nécessaires. Si tu as d'autres idées, tu peux les ajouter à la liste et les proposer à tes camarades.

1. Écrire la biographie de Daniel Da Barca en la situant dans son contexte historique.

2. Réaliser un reportage sur les conditions de vie des prisonniers politiques dans les prisons franquistes en se basant sur les données fournies par le roman et pouvant être complétées par d'autres sources.

3. Filmer un entretien avec Marisa Mallo où elle parle sur sa relation avec Daniel Da Barca, celui qui a fini par être son mari.

4. Publier une brève anthologie commentée des meilleurs passages du roman en vue de promouvoir sa commercialisation.

5. Préparer le texte d'une pièce de théâtre basée sur le roman. 


\section{Le point de vue des étudiants}

33 Nous avons demandé l'avis des étudiants sur la séquence précédente moyennant une évaluation informelle sous forme de bref commentaire. En général, les activités ont été jugées « motivantes ", « innovantes », « bonnes », « intéressantes » et les étudiants ont dit avoir eu du plaisir ; ils ont ajouté qu'elles ont éveillé leur intérêt.

Certains ont estimé que le matériel fourni en classe - la critique littéraire, la quatrième de couverture, le texte informatif sur la guerre civile en Galice et la lecture du premier chapitre du roman, notamment - était «insuffisant» pour rédiger un reportage « complet et élaboré » et ils semblent regretter d'avoir dû puiser dans des ressources supplémentaires (Internet) pour compléter l'information contextuelle et pouvoir écrire leur reportage. Certains d'entre eux, par contre, ont ressenti cette « insuffisance » comme un vrai atout pour chercher à en savoir plus et pour avoir la motivation de s'engager dans la lecture du roman.

Les commentaires sur la rédaction du reportage mettent en évidence que cette activité a été perçue de façon valorisante par les apprenants. Bien qu'un peu anecdotique, l'un d'entre eux signale, par exemple : «j'ai appris à écrire un reportage bien que j'avais déjà une idée de comment le faire. Écrire ce reportage m'a servi personnellement pour me montrer à moi-même que je pourrais être un bon journaliste, si je voulais me consacrer à cette profession ».

Enfin, quant aux activités comme hameçon pour la lecture du roman, un apprenant exprime : «le matériel présenté était juste insuffisant pour nous persuader de lire le livre entier, ce que je commencerai à faire dès aujourd'hui même »; et un autre : "[n]'importe quelle information qu'on puisse fournir avant la lecture d'un livre peut être utile et elle peut nous emmener à lire le livre ou à ne pas le lire. Dans mon cas, j'ai été convaincu. Je commencerai à le lire immédiatement ». Et encore : «bien sûr que j'aimerais lire le livre parce que ça me permettrait d'apprendre davantage sur ce qui s'est passé en cette époque de guerre et de conflits $"$.

On doit noter que l'intérêt lié à la découverte d'un pan de l'histoire de l'Espagne, la guerre civile espagnole et l'après-guerre, est mentionné à maintes reprises et valorisé, ce qui souligne l'importance qu'a pour les apprenants de lire et d'écrire sur des sujets qui véhiculent des vrais contenus, susceptibles de les interpeller: ces activités «m'[ont] ouvert les yeux sur la dureté de la guerre civile pour l'Espagne, je n'avais pas beaucoup d'information sur cette période ", écrit un des apprenants.

\section{Conclusion}

Ce rapide parcours au travers de quelques façons d'approcher la lecture littéraire en LE a permis d'esquisser des pistes visant à stimuler l'implication des apprenants, notamment par leur participation dans le choix du texte, par la prise en compte de leurs possibles difficultés face au fait littéraire, par l'intégration de la dimension personnelle, subjective et émotive, par l'interrogation du texte, par l'élaboration d'hypothèses de lecture et, enfin, par la manipulation du texte moyennant l'écriture. Lors de la lecture des textes littéraires en LE nous avons tout intérêt en tant qu'enseignants à ce que nos élèves expriment leurs réponses face au littéraire et à valoriser leurs opinions avec des activités qui permettent la discussion, le partage d'information et la manipulation des textes. Ce 
sont des moyens d'amener les apprenants non seulement à mieux se familiariser avec ceux-ci et, ensuite, à mieux les appréhender, mais aussi à le faire tout en construisant ses propres compétences linguistiques et culturelles en LE.

\section{BIBLIOGRAPHIE}

\section{Bibliographie}

Albert, M-C. \& Souchon, M. (2000). Les textes littéraires en classe de langue. Paris : Hachette.

Baroni, R. \& Rodriguez, A. (éds.) (2014). Les passions en littérature. Lausanne : Université de Lausanne, Études des Lettres.

Bemporad, Ch. (2014). « Lectures et plaisirs : pour une reconceptualisation des modes et des types de lecture littéraire ». In Baroni, R. \& Rodriguez, A. (éds.). Les passions en littérature. Lausanne : Université de Lausanne, Études des Lettres, pp. 65-83.

Bemporad, Ch. \& Jeanneret, Th. (éds.) (2007). Lectures littéraires et appropriation des langues étrangères. Lausanne : Université de Lausanne.

Conseil de l'Europe (2001): Cadre européen commun de référence pour les langues. Paris : Les Éditions Didier.

Cornaire, C. (1991). Le point sur la lecture. Paris : Clé International.

David, J. (2014). « Chloroforme et signification ». In Baroni, R. \& Rodriguez, A. (éds.). Les passions en littérature. Lausanne : Université de Lausanne, Études des Lettres, pp. 19-32.

Le Goff, F. (2011). « Les malles du lecteur, ou la lecture en écrivant ». In Mazauric, C., Fourtanier, M.J. \& Langlade, G. (éditeurs) (2011). Textes de lecteurs en formation, Bruxelles : Peter Lang, pp. 219-229.

Mazauric, C., Fourtanier, M.J. \& Langlade, G. (éditeurs) (2011 a). Le Texte du lecteur. Bruxelles : Peter Lang.

Mazauric, C., Fourtanier, M.J. \& Langlade, G. (éditeurs) (2011 b). Textes de lecteurs en formation, Bruxelles : Peter Lang.

Pendanx, M. (1998). Les activités d'apprentissage en classe de langue. Paris : Hachette.

Ricardou, J. (1990). « Deviens, lecteur, le scripteur que tu es ». Pratiques 67. pp.105-125.

Rivas, M. (2006). El lápiz del carpintero. Madrid: Santillana.

Rouxel, A. \& Langlade G. (2004). Le Sujet lecteur. Lecture subjective et enseignement de la littérature. Rennes : Presses Universitaires de Rennes.

Tauveron, C. (2002). Lire la littérature à l'école. Paris : Hatier. 


\section{Sitographie}

Blog littéraire de Carmina, De tinta en vena: http://detintaenvena.blogspot.ch/2011/01/el-lapizdel-carpintero-manuel-rivas.html, consulté en septembre 2015.

Discours de M. Vargas Llosa à l'occasion de sa nomination comme Docteur Honoris Causa par l'Université de Salamanca : http://cultura.elpais.com/cultura/2015/09/17/ actualidad/1442500868_851297.html, consulté en octobre 2015.

\section{NOTES}

1. Le discours où se trouvent ces réflexions a été prononcé lors de la nomination de Vargas Llosa comme Docteur Honoris Causa à l'Université de Salamanca et il disponible dans: http:// cultura.elpais.com/cultura/2015/09/17/actualidad/1442500868_851297.html, consulté en octobre de 2015.

2. Pour une réflexion sur le rôle des lectures littéraires dans l'appropriation des langues étrangères, voir M.-C. Albert \& M. Souchon (2000) et Ch. Bemporad \& T. Jeanneret (2007).

3. Voir C. Cornaire (Cornaire, 1991: 47 et suivantes) pour une synthèse sur les difficultés de lecture en L2, et aussi M. Pendanx (Pendanx, 1998 : 79-107) pour les activités de compréhension et de réception en $\mathrm{L} 2$.

4. Il est utile de signaler que certains personnages sont déjà introduits dans le tableau ; il s'agit d'un personnage collectif (les prisonniers), d'un personnage secondaire (Zalo Puga) et d'un objet (le crayon du charpentier) qui jouera un rôle primordial dans le développement de l'histoire au point de pouvoir être considéré comme un personnage à part entière. Ils apparaissent tous dans la critique ou dans la quatrième de couverture données aux élèves mais, étant à peine mentionnés, ils pourraient passer inaperçus.

5. «El principio del libro me aturdió un poco, me descentró y tuve que volver a empezar su lectura, y llegado al mismo punto me volví a perder de nuevo dentro de la historia, y eso se debe al distinto enfoque de narración que se produce de forma brusca y sin previo aviso. » (http:// detintaenvena.blogspot.ch/2011/01/el-lapiz-del-carpintero-manuel-rivas.html, consulté le 10 septembre de 2015).

6. «Este libro además de entretenerme y deleitarme con la forma en que está escrito me ha servido para profundizar en la guerra civil, en sus odios y en sus secretos... [...] He descubierto muchas cosas que antes no sabía. [...]_He sentido empatía por el doctor da Barca y menos por Herbal porque es un hombre tosco y malvado, con alguna pincelada de bondad que la envidia esconde en multitud de ocasiones.»_(http://detintaenvena.blogspot.ch/2011/01/el-lapiz-delcarpintero-manuel-rivas.html, consulté le 10-10-2015).

7. Voir Ch. Bemporad (Bemporad, $2014: 67-72$ ).

\section{RÉSUMÉS}

En partant des écueils particuliers de la lecture littéraire en LE, cet article propose quelques ressources pour faciliter l'accès à la lecture en $L E$ de textes littéraires longs, tels les romans, à des apprenants ayant un niveau B1 confirmé ou un niveau B2. La possibilité de choisir le texte à lire, le dialogue entre texte et lecteur 
par le biais de l'écriture ou la lecture extensive sont proposés, entre autres, comme des voies motivantes et facilitatrices pour accéder au texte littéraire. Les propositions sont illustrées par quelques exemples tirés d'une séquence didactique conçue pour la lecture du roman $\mathrm{El}$ lápiz del carpintero de l'écrivain espagnol Manuel Rivas dans une classe d'espagnol LE.

Taking into account the difficulties of reading litterature in $F L$, this article proposes some ressources aimed to facilitate the reading of long litterary texts (such as novels) in FL to students having a confirmed B1 or a B2 level. The possibility of choosing the text to be read, the dialogue between text and reader through writting, or free reading are presented, among other activities, as motivating and easing ways to have access to litterary texts in FL. Propositions are illustrated by some examples taken from a didactic sequence created to read the novel El lápiz del carpintero by the Spanish writer Manuel Rivas in a class of Spanish as a foreign language.

INDEX

Mots-clés : didactique des langues étrangères, lecture littéraire, écriture, didactisation de El lápiz del carpintero, Rivas Manuel

Keywords : foreign languages didactics, reading literature, writing, didactics of El lápiz del carpintero, Rivas Manuel

\section{AUTEUR}

\section{VICTORIA BÉGUELIN-ARGIMÓN}

